\title{
Relationship between potential barriers to early mobilization in adult patients during intensive care stay using the Perme ICU Mobility score
}

\author{
Esther C. Wilches Luna PT, MSc $c^{1,2,3,4}$, Christiane Perme PT, $\mathrm{CCS}^{5}$, Ada Clarice Gastaldi PT, PhD ${ }^{4}$
}

\begin{abstract}
EC Wilches Luna, C Perme, AC Gastaldi. Relationship between potential barriers to early mobilization in adult patients during intensive care stay using the Perme ICU Mobility score. Can J Respir Ther 2021;57:148-153. doi: 10.29390/cjrt-2021-018.
\end{abstract}

\begin{abstract}
Background: Identifying barriers to early mobilization is essential for the management of patients in the intensive care unit (ICU). Our objective was to identify the potential barriers to early mobilization in adult patients using the Perme ICU Mobility Score (Perme Score) and its relationship with days of mechanical ventilation (MV) and length of stay in ICU.

Methods: This was a pilot, observational, and prospective study. We included 142 adult patients admitted to a 14-bed ICU, in a fourth-level complexity hospital in Cali, Colombia. The Perme Score was used to evaluate potential barriers to mobility. We used the Spearman's correlation coefficient to find potential correlations between the number of barriers to mobility per patient and the duration of MV and ICU stay.

Results: We identified significant inverse correlations between total days in MV and the total score of barriers to mobility at ICU admission $(r=-0.773$; $p<0.05)$ and at ICU discharge $(r=-0.559 ; p<0.05)$. Also, between ICU length of stay and total score of barriers to mobility at ICU admission $(r=-0.420$; $p<0.05)$ and at ICU discharge $(r=-0.283 ; p<0.05)$. Moreover, we found a significant correlation between total score of the barriers item and total Perme score $(r=0.91 ; p<0.01)$.

Conclusions: Using the Perme Score we identified potential barriers to mobility upon admission to the ICU that were maintained until discharge. Our findings indicate a strong positive correlation at ICU admission between the total Perme Score and the total score of "Category \#2 - Potential Mobility Barriers" in the Perme Score.
\end{abstract}

Key Words: critical illness; early ambulation; intensive care units; respiration, artificial; hospitals

\section{INTRODUCTION}

Understanding barriers to early mobilization (EM) is crucial for the management of patients in the intensive care unit (ICU). Growing evidence supports the use of EM activities in the ICU as a strategy to improve functional recovery during and after prolonged critical illness, reduce the effects of intensive care acquired muscle weakness (ICUAW), as well as reduce hospitalization days and incidence of delirium [1, 2]. ICUAW is a process of multifactorial etiology defined as muscle weakness that develops after the onset of critical illness [1]. This process increases the duration of mechanical ventilation (MV), hospitalization, mortality, and morbidity [1-4], and its effect may be greater in those patients who require invasive $\mathrm{MV}$, even in periods of less than 7 days [1].

EM is defined as mobility activities that start immediately after physiological stabilization: neurological, respiratory, and cardiovascular, generally within $24-48 \mathrm{~h}$ of admission to the ICU $[1,5-8]$. Although the benefits of EM are well reported in the literature, some authors have identified the presence of barriers that limit its implementation. Dubb et al. [9] described patient-related barriers to EM such as the presence of catheters, drainage tubes, tubes, deep sedation practices, the variability of vascular accesses, the lack of equipment and a coordinated multidisciplinary team, and the potential increase in workload [9]. Parry et al. [8] suggested instruments to assess the mobility of patients in ICU such as Physical Function in Intensive Care Test-score, Functional Status Score for the ICU, Chelsea Critical Care Physical Assessment Tool, ICU Mobility Scale, Surgical Intensive Care Unit Optimal Mobility Scale, Perme Intensive Care Unit Mobility Score (Perme Score), Acute Care Index of Function, Critical Care Functional Rehabilitation Outcome Measure, De Morton Mobility Index, Short Physical Performance Battery, and Manchester Mobility Score [8].

When considering all the aforementioned mobility instruments, the Perme Score is the only tool that includes a detailed category with four different items that specifically identify the potential barriers when mobilizing patients. There are different types of barriers such as patient-related, structural, cultural, and process-related [10], but the

\footnotetext{
${ }^{1}$ Exercise and Cardiopulmonary Health Research Group (GIESC by its Spanish acronym) Universidad del Valle, Cali, Colombia

${ }^{2}$ Faculty of Health, School of Human Rehabilitation, Universidad del Valle, Cali, Colombia

${ }^{3}$ Society of Respiratory Physiotherapists (SOFIRE SAS by its Spanish acronym), Cali, Colombia

${ }^{4}$ Department of Health Sciences, Ribeirão Preto Medical School, University of São Paulo, Ribeirão Preto, São Paulo / Brazil

${ }^{5}$ Department Rehabilitation Services, Houston Methodist Hospital. Houston, TX, United States of America

Correspondence: Esther Cecilia Wilches-Luna, Universidad del Valle, $3^{a} \# 36$ b-05 Edificio Perlaza, Second floor, Cali, Colombia. Tel: +57 3174374624 ,

E-mail: esther.wilches@correounivalle.edu.co
}

Published online at https://www.cjrt.ca 22 November 2021

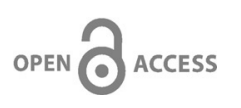

This open-access article is distributed under the terms of the Creative Commons Attribution Non-Commercial License (CC BY-NC) (http:// creativecommons.org/licenses/by-nc/4.0/), which permits reuse, distribution and reproduction of the article, provided that the original work is properly cited and the reuse is restricted to noncommercial purposes. For commercial reuse, contact editor@csrt.com 
potential barriers item in the Perme Score only relates to patient-related barriers. The Perme Score has high inter- and intra-rater reliability and has been translated and culturally adapted to different languages such as Portuguese [11], German [12], and Spanish [13]. In clinical practice, it is important to recognize the burden of potential barriers to mobility activities in the ICU to facilitate safe and timely implementation of a mobility program [9]. Since the Perme Score is the only mobility tool that considers the measurement of potential mobility barriers, the main objective of this study was to explore the relationship between the "Category \#2 - Potential Mobility Barriers" in the Perme Score with duration of MV and length of stay (LOS) in ICU. A secondary objective was to look for a potential correlation between the total score of the Category \#2 - Potential Mobility Barriers and the total score of the Perme Score.

\section{MATERIALS AND METHODS}

The Human Ethics Committee of the Universidad del Valle approved the study [\# 011-015] and informed consent was obtained from patients or family members according to local regulations. This was a pilot, observational, and prospective study.

\section{Settings}

The patient population considered for this study were adult patients admitted to a 14-bed ICU, in a fourth-level complexity hospital in the city of Cali, Colombia. A convenience sample of 142 patients was used, given that this size is larger than necessary for a mean difference with $90 \%$ power and $95 \%$ confidence [14]. The recruitment period was from November 2016 to July 2017.

\section{Participants}

Patients were age 18 years or older, willing to participate in the study, and classified as level of independent functionality before admission to the general medical and surgical ICU (Barthel index above 90-reported by the family or patient based on the health situation seven days before admission to the ICU). Patients with hearing impairment, who did not speak Spanish, were transferred from other units, were readmitted to the ICU, or had a LOS in ICU greater than 21 days, were excluded.

\section{Variables and data sources}

\section{Description of the Perme Score}

The "Perme Intensive Care Unit Mobility Score" (Perme Score) contains 15 items grouped into seven categories: mental status, potential barriers to mobility, functional strength, mobility in bed, transfers, gait, and endurance [7]. The total Perme Score varies from 0 to 32 and has a maximum range of 2 to 4 points for each of the 15 items. A high total Perme Score indicates good mobility and few barriers to EM. Supplemental material $1^{1}$ shows the Category \#2 - Potential Mobility Barriers related to the patient items from the Perme Score.

\section{Pilot study}

The four physiotherapists who oversaw data collection had a $12-\mathrm{h}$ training session in the use of the Perme Score. The physiotherapists evaluated each patient, and the duration, execution, comments, and questions generated during the process were recorded. Once the training was concluded, a pilot study was conducted with a convenience sample of 30 patients who were not included in the final sample of the study.

Two pairs of trained study evaluators were formed (each pair had a specialist physiotherapist with a postgraduate degree in intensive care and more than five years of experience, and a junior physiotherapist an undergraduate degree and more than five years of experience), and they independently assessed each patient at the same time with the Perme Score twice, exchanging their roles in the second assessment. For each pair of physiotherapists who gathered the data, letters A and B were

\footnotetext{
${ }^{1}$ Supplementary material are available on the journal website at https:// www.cjrt.ca/wp-content/uploads/Supplemental-informationCJRT-2021-18.docx
}

assigned. Therapist "A" evaluated the patient, and therapist "B" observed the entire process without physically touching the patient and was responsible for collecting the other study variables such as: sociodemographic data, medical diagnosis, MV time, Apache II score (Acute Physiology and Chronic Health Evaluation II), level of sedation, time of stay in ICU, type of weaning, and place of discharge. Each pair of evaluators independently applied the Perme Score simultaneously for the same patient, and filled out the data collection form, after completing the Perme Score and before initiating any physiotherapy interventions performed by the hospital. The application of the two scales was carried out at admission to the study and at discharge from the ICU.

The physiotherapists were randomized with a balanced incomplete blocks design, using sealed envelopes. The main researcher and the ICU work team were masked. Before applying the Perme Score, we used the institution's standardized EM checklist, addressing the indications, hemodynamic, neurologic, and motor stability.

\section{Statistical analysis}

The Perme Scores for each patient reported by both evaluators were entered in a database. Comparisons were made to identify differences between the recorded data (inter-rater reliability: intraclass correlation coefficient 0.99 and 1.00 in the two measured moments). We used the measures of central tendency, mean, and median for the description of the clinical characteristics of the patients. For the measure of dispersion according to the type and distribution of each variable, we used the standard deviation and absolute frequencies and percentages. We calculated the frequency for each of the potential barriers to EM, both at admission and at discharge of ICU patients. We used the nonparametric WilcoxonMann-Whitney test to identify significant differences between barriers to mobility at admission and discharge. The Spearman's correlation coefficient was used to identify potential correlations between the number of barriers to mobility per patient and the duration of MV and ICU stay. It was also used to identify potential correlation between the Category \#2 - Potential Mobility Barriers score and the total Perme Score. Values close to 1 indicate a strong and positive correlation; values close to -1 indicate a strong and negative correlation; values close to zero indicate that there is no linear correlation [15]. We used the software SPSS 22 for all the statistical analyses.

\section{RESULTS}

We included data from 142 patients in the study. Figure 1 shows the recruitment and flow of participants. Over a five-month period, 354 patients were admitted to the ICU; 204 met exclusion criteria.

The clinical characteristics of the patients are described in Table 1 . Seventy-three patients were male (51.4\%) and 69 were female $(48.6 \%)$, and the average age was $58 \pm 17$ years $( \pm$ SD). There were $57.8 \%$ of patients hospitalized for clinical reasons, heart conditions being the most prevalent reason for hospitalization. Invasive MV was present in $39.4 \%$ of the cases and easy weaning, defined as successful extubation after a single spontaneous breathing trial, occurred in $91 \%$ of the patients. The percentages of identified patients with potential barriers to EM is shown in Table 2. According to the item of potential barriers to EM in the Perme score, MV, pain, and two or more devices were identified as potential barriers to mobility both at admission and at discharge, although they presented statistical significance $(p=0.000)$. The presence of intravenous drips was also identified as a barrier to both admission and discharge without statistical significance.

Arterial lines and urinary catheters were the medical devices most reported upon admission and discharge from the ICU, as shown in Table 3. Upon admission to the ICU, a Category \#2 - Potential Mobility Barriers total score of zero was identified in $37 \%$ of patients, and a score of three in $13 \%$ of the patients. At ICU discharge, $57 \%$ of patients had a score of two, and $31 \%$ had a score of three. The maximum score of four was not reached in either of the two data collection times (Table 4). The relationship between the Category \#2 - Potential Mobility Barriers at admission and discharge compared with the duration of MV and ICU LOS was negative and statistically significant (Spearman's Rho $p<0.05$; Table 5). Additionally, regarding the relationship between the total 


\section{FIGURE 1}

Flow diagram of recruitment of participants.

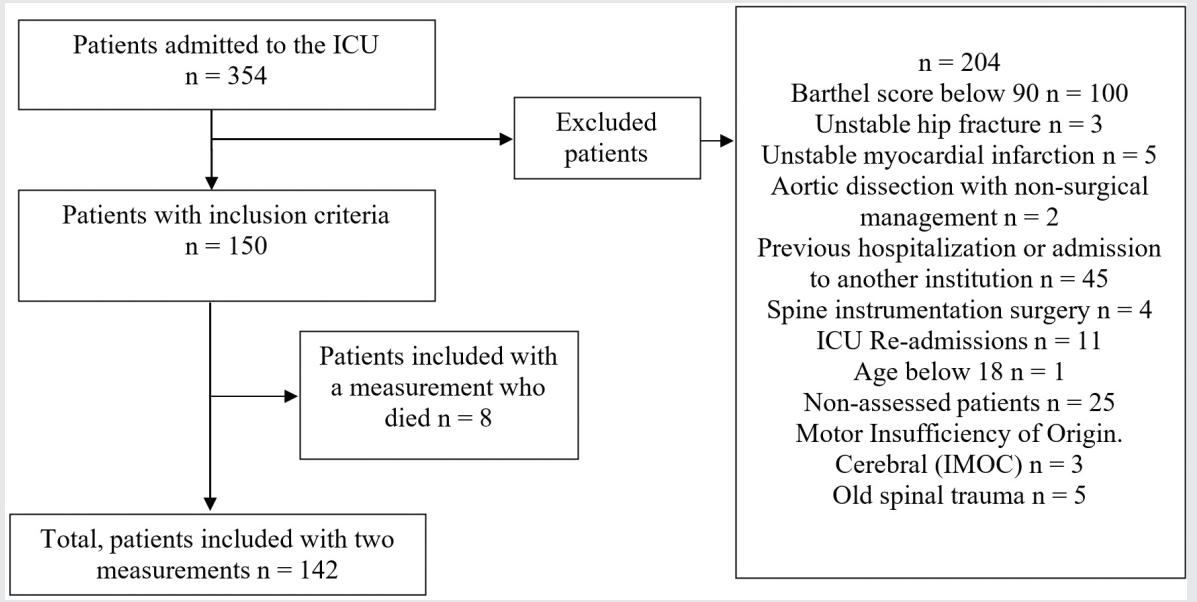

TABLE 1

Clinical characteristics of the patients

\begin{tabular}{|c|c|}
\hline Variables & $\begin{array}{l}\text { Sample data } \\
n=142\end{array}$ \\
\hline Age (mean $\pm S D)$ & $58.1 \pm 16.8$ \\
\hline Male, $n(\%)$ & $73(51.4)$ \\
\hline Female, $n(\%)$ & $69(48.6)$ \\
\hline Clinical reason for hospitalization & $n=82$ \\
\hline Cardiac, $n(\%)$ & $39(27.5)$ \\
\hline Respiratory, $n(\%)$ & $16(11.3)$ \\
\hline Gastrointestinal, $n(\%)$ & $9(6.3)$ \\
\hline Neurological, $n(\%)$ & $1(0.7)$ \\
\hline Sepsis, $n(\%)$ & $5(3.5)$ \\
\hline Other, $n(\%)$ & $12(8.5)$ \\
\hline Surgical reason for hospitalization & $n=60$ \\
\hline Cardiac, $n(\%)$ & $33(23.2)$ \\
\hline Neurosurgical, $n(\%)$ & $17(12)$ \\
\hline Trauma, $n(\%)$ & $3(2.1)$ \\
\hline Other, $n(\%)$ & $7(4.9)$ \\
\hline Invasive mechanical ventilation, $n(\%)$ & $56(39.4)$ \\
\hline Noninvasive mechanical ventilation, $n(\%)$ & $3(2.1)$ \\
\hline Tracheostomy, $n(\%)$ & $6(4.2)$ \\
\hline Time (days) of mechanical ventilation, (Mean $\pm \mathrm{SD}$ ) & $2.2 \pm 5.4$ \\
\hline Type of weaning & $n=60$ \\
\hline Easy weaning, $n(\%)$ & $51(91)$ \\
\hline Prolonged weaning, $n(\%)$ & $5(8.9)$ \\
\hline Weaning time (days), median $(\mathrm{IQR})^{* *}$ & $1(0-1)$ \\
\hline Total time (days) in intensive care unit (Mean $\pm \mathrm{SD}$ ) & $4.3 \pm 5.8$ \\
\hline Discharge from ICU & $n=142$ \\
\hline Intermediate care unit, $n(\%)$ & $119(83.8)$ \\
\hline Hospitalization rooms, $n(\%)$ & $9(6.3)$ \\
\hline Other, $n(\%)$ & $14(9.9)$ \\
\hline \multicolumn{2}{|l|}{ Medications } \\
\hline Sedatives, $n(\%)$ & $25(17.6)$ \\
\hline Analgesics, $n(\%)$ & $25(17.6)$ \\
\hline Muscle relaxants, $n(\%)$ & $3(2.1)$ \\
\hline *APACHE II, Median (IQR) & $14.6(9.9-26.2)$ \\
\hline
\end{tabular}

*APACHE, Acute Physiology and Chronic Health Evaluation - Measured in 95 patients; IQR, interquartile range; SD, standard deviation.
TABLE 2

Potential barriers to early mobilization with the Perme Score $(n=142)$

\begin{tabular}{lcccccc}
\hline Barrier & \multicolumn{2}{c}{ Admission } & & \multicolumn{2}{c}{ Discharge } & \\
\cline { 2 - 3 } & $\boldsymbol{n}$ & $\%$ & & $\boldsymbol{n}$ & $\%$ & $\boldsymbol{p}^{*}$ \\
\hline $\begin{array}{l}\text { Is the patient on } \\
\text { mechanical ventilation or } \\
\text { noninvasive ventilation? }\end{array}$ & 59 & 42 & & 6 & 4 & 0.000 \\
$\begin{array}{l}\text { Pain } \\
\text { The patient has two or }\end{array}$ & 85 & 60 & & 19 & 13 & 0.000 \\
$\begin{array}{l}\text { more devices } \\
\text { Other }\end{array}$ & 118 & 84.3 & & 93 & 66.4 & 0.000 \\
Intravenous drips & 43 & 30.7 & & 34 & 24.3 & 1.00 \\
\hline
\end{tabular}

*Statistical significance $p<0.05$.

Perme Score with the total score for the Category \#2 - Potential Mobility Barriers, we identified a significant positive relationship upon admission to the ICU $(r=0.91 ; p<0.01)$.

\section{DISCUSSION}

In this study, our objective was to identify the potential barriers to mobility in patients in ICU by using the Perme Score and highlight the importance of recognizing mobility barriers as part of the strategies that facilitate EM implementation. The item that differentiates the Perme Score from other available mobility scales is the Category \#2 - Potential Mobility Barriers, which includes pain, the use of MV, medical devices, vascular accesses, and the presence of intravenous infusions. According to our results regarding these barriers, patient-related mobility barriers remained present throughout the entire ICU stay, and therefore physical assistance was required for any mobility activity. We also believe that it is important not only to identify mobility barriers, but to also generate and implement strategies to minimize the impact of these barriers.

Barber et al. [16], in a qualitative descriptive study with focus groups, studied the multidisciplinary perceptions of clinical staff (physicians, nurses, and physiotherapists) have related to the barriers and facilitators of mobility in the ICU environment. In this study, three relevant issues emerged regarding barriers to mobilization: culture, communication, and lack of resources [16]. For Hodgson et al. [17], EM barriers and facilitators can be divided into patient factors, ICU team factors, and organizational factors. The Category \#2 - Potential Mobility Barriers in the 
TABLE 3

Medical devices

\begin{tabular}{|c|c|c|c|c|c|}
\hline \multirow{2}{*}{ Devices } & \multicolumn{2}{|c|}{ Admission } & \multicolumn{2}{|c|}{ Discharge } & \multirow{2}{*}{$p^{*}$} \\
\hline & $n$ & $\%$ & $n$ & $\%$ & \\
\hline Supplemental oxygen & 43 & 30 & 40 & 28 & 0.680 \\
\hline Urinary catheter & 79 & 56 & 53 & 37 & 0.000 \\
\hline Endotracheal tube & 49 & 35 & 2 & 1 & 0.000 \\
\hline Tracheostomy & 0 & 0 & 6 & 4 & 0.014 \\
\hline $\begin{array}{l}\text { Peripherally inserted } \\
\text { central catheter }\end{array}$ & 6 & 4 & 3 & 2 & 0.180 \\
\hline Arterial line & 88 & 62 & 42 & 30 & 0.000 \\
\hline Dialysis catheter & 6 & 4 & 6 & 4 & 1.000 \\
\hline Nasogastric tube & 23 & 16 & 6 & 4 & 0.000 \\
\hline Chest tube & 30 & 2 & 26 & 18 & 0.206 \\
\hline Temporary pacemaker & 4 & 3 & 1 & 1 & 0.046 \\
\hline Pulmonary artery catheter & 1 & 1 & 0 & 0 & 0.317 \\
\hline $\begin{array}{l}\text { Patient-controlled epidural } \\
\text { analgesia }\end{array}$ & 1 & 1 & 2 & 1 & 0.317 \\
\hline $\begin{array}{l}\text { Continuous renal } \\
\text { replacement therapy }\end{array}$ & 0 & 0 & 1 & 1 & 1.000 \\
\hline $\begin{array}{l}\text { Wound with closed } \\
\text { suction system }\end{array}$ & 1 & 1 & 2 & 1 & 0.317 \\
\hline 2 or more / other & 49 & 35 & 35 & 25 & 1.000 \\
\hline
\end{tabular}

${ }^{*}$ Statistical significance $p<0.05$.

Perme Score includes barriers related to the patient, which is a particularly important characteristic since it allows the physiotherapist to identify important aspects that must be considered to improve the results of EM.

Previous studies by Nydahl et al. [18] in Germany and Berney et al. [19] in Australia and New Zealand have reported a low prevalence of EM mainly in patients with $\mathrm{MV}$, which has also been observed in the Latin American population (Colombia and Brazil) by Wilches-Luna et al. [20] and Fontela et al. [21]. In the present study, upon admission to the ICU, the presence of MV was identified in $42 \%$ of the patients as a potential barrier to EM according to the Perme Score. These results are consistent with the available evidence that reports a low prevalence of EM out of bed in patients with MV $[19,21]$. Our results are also consistent with those of Hodgson et al. [17] regarding the resistance of ICU physicians to mobilize patients with MV support, despite scarcity of reported adverse events and the potential benefits of EM. Here, we confirm the significant differences regarding the presence of MV as a potential barrier to EM upon admission versus discharge from the ICU.

Although studies support that EM is safe and feasible in ventilated patients, it is not yet viewed as a standard of care in clinical practice [16, $19,21]$. This could be influenced by the presence of other barriers, such as the need for more education, insufficient staff, resources, and culture of EM [16, 19, 21]. The implementation of an EM protocol must include the identification of both perceived and real barriers [4, 22]. Dubb et al. [9] conducted a systematic review with the objective of identifying barriers to EM and discussed strategies to overcome them. The results showed that vascular access devices, tubes and drains were identified as barriers in $45 \%$ of the studies that mentioned patient-related barriers. In the present study, upon ICU admission and discharge, arterial lines (62\% vs. $30 \%$ ) and urinary catheters (56\% vs. $37 \%$ ) were identified as barriers to EM. The use of arterial vascular access constitutes a fundamental aspect of the care of the critical patient due to the information it provides about the cardiocirculatory physiopathology, and it will be present in most patients [23]. Therefore, strategies must be established to maintain adequate management of these devices to facilitate EM, with relatively simple changes in management, such as the placement selection for vascular
TABLE 4

"Category \#2 - Potential Mobility Barriers" scores

\begin{tabular}{lcccccc}
\hline Total score of the item & \multicolumn{2}{c}{ Admission } & & \multicolumn{2}{c}{ Discharge } \\
\cline { 2 - 3 } \cline { 5 - 6 } $\begin{array}{l}\text { Potential Barriers to Early } \\
\text { Mobilization }\end{array}$ & $\boldsymbol{n}$ & $\%$ & & $\boldsymbol{n}$ & $\%$ \\
\hline 0 & 53 & 37 & & 4 & 3 \\
1 & 33 & 23 & & 13 & 9 \\
2 & 38 & 27 & & 81 & 57 \\
3 & 18 & 13 & & 44 & 31 \\
4 & 0 & 0 & & 0 & 0 \\
\hline
\end{tabular}

TABLE 5

Relationship between mechanical ventilation - ICU stay with the total score of the item potential barriers to EM of the Perme Score

\begin{tabular}{lcc}
\hline Variables & $\begin{array}{c}\text { Spearman's } \\
\text { Rho }\end{array}$ & $p$-value \\
\hline $\begin{array}{l}\text { Admission to ICU vs. days of mechanical } \\
\text { ventilation }\end{array}$ & $-0.773^{* *}$ & 0.000 \\
$\begin{array}{l}\text { ICU discharge vs. days of mechanical } \\
\text { ventilation }\end{array}$ & $-0.559^{* *}$ & 0.000 \\
$\begin{array}{l}\text { Admission to ICU vs. days of stay in ICU } \\
\text { ICU discharge vs. days of stay in ICU }\end{array}$ & $-0.420^{* *}$ & 0.000 \\
\hline
\end{tabular}

Note: Variables is the total score of the item's possible barriers. EM, early mobilization; ICU, intensive care unit.

${ }^{* *}$ Statistical significance $p<0.05$.

access devices [23]. These types of strategies require a change in culture and communication among the interdisciplinary team.

Regarding the intravenous infusions, specifically in the use of vasoactive pharmacological agents, the evidence indicates that it should not be an absolute contraindication for EM [24]. However, to date, the specific doses or changes in the doses that must be considered when performing EM have not been defined [23]. We identified in this study that, at ICU admission and discharge, the presence of intravenous infusions was reported as a potential barrier to EM according to the Perme Score. In view of intravenous infusions being routinely used in critical illness, we consider that identifying them as barriers to EM early on can lead to discussions amongst ICU health professionals. Once the risks have been detected, measures should be implemented to work safely and reduce adverse events during EM, helping to improve the quality of care and preventing unnecessary risks associated with prolonged bedrest.

Pain assessment in critically ill patients is frequently undervalued. Asking the patient is the best way to assess pain, considering that it is a subjective sensation and, therefore, the sensations reported by the patient are the basis for making decisions [25]. In this context, the Perme Score considers pain as a potential barrier if the patient is unable to determine it or indicates the presence of pain. In the results of our study, pain was identified as a barrier in $60 \%$ of the patients upon admission to ICU and decreased to $13 \%$ of the patients at discharge with statistical significance $(p=0.000)$. These findings indicate the need for appropriate interdisciplinary approach to pain assessment and management, considering that untreated pain in critical patients can interfere with EM practices and become a barrier throughout the ICU stay. Chamorro et al. [26] highlighted the need to properly monitor and treat pain and, if indicated, to keep the patient awake during MV and perform daily trials of spontaneous ventilation and EM. They also explained the importance of developing analgesia and sedation protocols to be utilized before or during mobilization and when performing diagnostic or therapeutic tests that may cause pain, anxiety, or fear to the patient [26].

The findings of the present study showed a moderate correlation between the total score of the Category \#2 - Potential Mobility Barriers 
in the Perme Score upon admission and discharge from the ICU with days of MV. The same relationship was also present with ICU LOS, suggesting that the lower the score of the Category \#2 - Potential Mobility Barriers items, the more barriers to mobility are identified.

In recent years, the hypothesis that immobilization is one of the main risk factors contributing to the pathogenesis and severity of ICUAW has been reinforced. Jolley et al. [27] found that up to $80 \%$ of patients admitted to the ICU develop some form of neuromuscular dysfunction. They also found that survivors of critical illness experience physical and cognitive deficiencies associated with the severity of the illness and the duration of stay in ICU, persisting for years after ICU discharge. ICUAW can cause a deterioration of functional capacity, limiting activities of daily life, restricting participation, and negatively impacting the quality of life of patients and their families [27]. Therefore, starting from ICU admission, we believe that it is important to identify the barriers that may limit the initiation of EM. This will facilitate the evolution of clinical recovery to determine the therapeutic priorities, and to choose the most appropriate EM strategy.

In this study, a strong positive correlation was identified at ICU admission between the total Perme Score ( 32 points) and the total score of Category \#2 - Potential Mobility Barriers in the Perme Score (4 points), a lower Category \#2 - Potential Mobility Barriers indicating greater barriers and lower mobility. These results are consistent with other studies that identified the presence of some of these barriers [28, 29], but that did not report the use of validated instruments for their evaluation. All the above information suggests that the use of a valid and reliable ICU mobility scale that facilitates the identification of barriers to EM might facilitate an early start of mobilization and functional recovery during critical illness $[4,28-30]$.

This study was limited by the single-center investigation, which does not allow extrapolation of the results, and by the lack of barriers classification by subgroups of patients. There are methodological strengths of the study related to having scores measured by two evaluators at two different times, which allowed for distinguishing potential barriers through the Perme Score at ICU admission and discharge.

\section{CONCLUSIONS}

Using the Perme Score, we identified potential barriers to mobility upon admission to the ICU, which continued until discharge. Our findings indicate a strong positive correlation at ICU admission between the total Perme Score and the total score of Category \#2 Potential Mobility Barriers in the Perme Score. Moreover, there was a negative relationship observed with the Category \#2 - Potential Mobility Barriers and the duration of MV and ICU LOS. We recommend carrying out additional studies that relate the potential barriers identified with the Perme Score with the start time and type of EM activities.

\section{DISCLOSURES}

\section{Disclosure of interest}

The authors declare that there is no conflict of interest regarding the publication of this article.

\section{Funding sources}

This study did not receive any specific grants from funding agencies in the public, commercial, or not-for-profit sectors.

\section{Ethical approval}

The Human Ethics Committee of the Universidad del Valle approved the study [\# 011-015] and informed consent was obtained from patients or family members according to local regulations.

\section{Contributors}

All authors were responsible for (i) substantial contributions to the conception and design of the work and the acquisition, analysis, and interpretation of data for the work; (ii) drafting the work and revising it critically for important intellectual content; (iii) final approval of the version to be published; and (iv) agreement to be accountable for all aspects of the work in ensuring that questions related to the accuracy or integrity of any part of the work are appropriately investigated and resolved. All authors read and approved the final version of the manuscript.

\section{REFERENCES}

1. Stevens RD, Marshall SA, Cornblath DR, et al. A framework for diagnosing and classifying intensive care unit-acquired weakness. Crit Care Med 2009;37(Suppl):S299-308. doi: 10.1097/CCM.0b013e3181b6ef67.

2. Morris PE, Goad A, Thompson C, et al. Early intensive care unit mobility therapy in the treatment of acute respiratory failure. Crit Care Med 2008;36:2238-43. doi: 10.1097/CCM.0b013e318180b90e.

3. Schweickert WD, Pohlman MC, Pohlman AS, et al. Early physical and occupational therapy in mechanically ventilated, critically ill patients: a randomised controlled trial. Lancet 2009;373:1874-82. doi: 10.1016/ S0140-6736(09)60658-9.

4. Hodgson CL, Berney S, Harrold M, Saxena M, Bellomo R. Clinical review: early patient mobilization in the ICU. Crit Care 2013;17:207-13. doi: $10.1186 /$ cc11820.

5. Martí Romeu JD. Debilidad muscular adquirida en la unidad de cuidados intensivos: ¿un problema con una única solución? [Intensive care unit-acquired muscular weakness: a problem with a single solution?]. Enferm Intensiva 2016;27(2):41-43. doi: 10.1016/j.enfi.2016.04.002.

6. Bailey P, Thomsen GE, Spuhler VJ, et al. Early activity is feasible and safe in respiratory failure patients. Crit Care Med 2007;35(1):139-45. doi: 10.1097/01.CCM.0000251130.69568.87.

7. Perme C, Nawa RK, Winkelman C, Masud F. A tool to assess mobility status in critically ill patients: the Perme Intensive Care Unit Mobility Score. Methodist Debakey Cardiovasc J 2014;10(1):41-9. doi: 10.14797/ mdcj-10-1-41.

8. Parry SM, Huang M, Needham DM. Evaluating physical functioning in critical care: considerations for clinical practice and research. Crit Care 2017;21(1):1-10. doi: 10.1186/s13054-017-1827-6.

9. Dubb R, Nydahl P, Hermes C, et al. Barriers and strategies for early mobilization of patients in intensive care units. Ann Am Thorac Soc 2016;13(5):724-30. doi: 10.1513/AnnalsATS.201509-586CME.

10. Alaparthi GK, Gatty A, Samuel SR, Amaravadi SK. Effectiveness, safety, and barriers to early mobilization in the intensive care unit. Crit Care Res Pract 2020;2020:1-14. doi: 10.1155/2020/7840743.

11. Kawaguchi YM, Nawa RK, Figueiredo TB, Martins L, Pires-Neto RC. Perme intensive care unit mobility score and icu mobility scale: translation into Portuguese and cross-cultural adaptation for use in Brazil. J Bras Pneumol 2016;42(6):429-34. doi: 10.1590/s1806-37562015000000301.

12. Nydahl P, Wilkens S, Glase S, et al. The German translation of the Perme Intensive Care Unit Mobility Score and inter-rater reliability between physiotherapists and nurses. Eur J Physiother 2018;20(2):10915. doi: 10.1080/21679169.2017.1401660.

13. Wilches-Luna EC, Hernández NL, de Oliveira AS, Kenji Nawa R, Perme C, Gastaldi AC. Perme ICU Mobility Score (Perme Score) and the ICU Mobility Scale (IMS): translation and cultural adaptation for the Spanish language. Colomb Med 2018;49(4):265-72. doi: 10.25100/ cm.v49i4.4042.

14. Wilches Luna EC, de Oliveira AS, Perme C, Gastaldi AC. Spanish version of the Perme Intensive Care Unit Mobility Score: minimal detectable change and responsiveness. Physiother Res Int 2021;26(1):e1875. doi: $10.1002 /$ pri. 1875 .

15. Akoglu H. User's guide to correlation coefficients. Turk J Emerg Med 2018;18(3):91-3. doi: 10.1016/j.tjem.2018.08.001

16. Barber EA, Everard T, Holland AE, Tipping C, Bradley SJ, Hodgson CL. Barriers and facilitators to early mobilisation in intensive care: a qualitative study. Aust Crit Care 2015;28(4):177-83. doi: 10.1016/j. aucc. 2014.11.001.

17. Hodgson CL, Capell E, Tipping CJ. Early Mobilization of patients in intensive care: organization, communication and safety factors that influence translation into clinical practice. Crit Care 2018;22(1):77. doi: 10.1186/s13054-018-1998-9.

18. Nydahl P, Ruhl AP, Bartoszek G, et al. Early mobilization of mechanically ventilated patients: a 1-day point-prevalence study in Germany. Crit Care Med 2014;42(5):1178-86. doi: 10.1097/CCM.0000000000000149.

19. Berney SC, Harrold M, Webb SA, et al. Intensive care unit mobility practices in Australia and New Zealand: a point prevalence study. Crit Care Resusc 2013;15(4):260-5. 
20. Wilches-Luna EC, Muñoz Arcos VE, Girón E, et al. Implementación de un programa de movilización temprana en pacientes críticos. Acta Colomb Cuid Intensivo 2015;15(4):287-92. doi: 10.1016/j.acci.2015.08.003.

21. Fontela P, Lisboa T, Forgiarini Jr LA, Friedman G. Early mobilization in mechanically ventilated patients: a one-day prevalence point study in intensive care units in Brazil. Clinics 2018;73:e241. doi: 10.6061/clinics/2018/e241.

22. Jolley SE, Regan-Baggs J, Dickson RP, Hough CL. Medical intensive care unit clinician attitudes and perceived barriers towards early mobilization of critically ill patients: a cross-sectional survey study. BMC Anesthesiol 2014;14:84. doi: 10.1186/1471-2253-14-84.

23. Koo KK, Choong K, Cook DJ, et al. Canadian Critical Care Trials Group. Early mobilization of critically ill adults: a survey of knowledge, perceptions and practices of Canadian physicians and physiotherapists. CMAJ Open 2016;4(3):E448-54. doi: 10.9778/cmajo.20160021.

24. Prasobh J, Surendran PJ, Muhamed Aleef EM, et al. Early mobilization of patients receiving vasoactive drugs in critical care units: a systematic review. JACPT 2021;12(1):37-48. doi: 10.1097/JAT.00000000000000140.
25. Pardo C, Muñoz T, Chamorro C. Monitorización del dolor. Recomendaciones del grupo de trabajo de analgesia y sedación de la SEMICYUC. Med Intensiva 2006;30(8):379-85. doi: 10.1016/ S0210-5691(06)74552-1.

26. Chamorro C, Romera MA. Dolor y miedo en la UCI [Pain and fear in the ICU]. Med Intensiva 2015;39(7):442-4. doi: 10.1016/j. medin.2015.05.005

27. Jolley SE, Bunnell AE, Hough C. ICU-acquired weakness. Chest 2016;150(5):1129-40. doi: 10.1016/j.chest.2016.03.045.

28. Dirkes SM, Kozlowski C. Early mobility in the intensive care unit: evidence, barriers, and future directions. Crit Care Nurse 2019;39(3):3342. doi: $10.4037 / \mathrm{ccn} 2019654$.

29. Leditschke IA, Green M, Irvine J, Bissett B, Mitchell IA. What are the barriers to mobilizing intensive care patients? Cardiopulm Phys Ther J 2012;23(1):26-29. doi: 10.1097/01823246-201223010-00005.

30. Hermans G, Van den Berghe G. Clinical review: intensive care unit acquired weakness. Crit Care 2015;19(1):274. doi: 10.1186/ s13054-015-0993-7. 